\title{
The Function of E6BP in Normal Human and HPV-Infected Cell Lines
}

\author{
Amanda Lima1, Anne Rietz ${ }^{1}$, Elliot A. Androphy ${ }^{1}$ \\ ${ }^{1}$ Department of Dermatology, Indiana University School of Medicine
}

\section{Background and Hypothesis:}

High-risk human papillomavirus type-16 and -18 cause approximately $70 \%$ of cervical and $60 \%$ of oropharyngeal cancers. While the majority of HPV infections are transient and resolve on their own, some infections persist and progress to cancer. The in vivo and in vitro high-risk HPV transformation ability works to immortalize primary human keratinocytes resulting from the activities of the viral oncoproteins E6 and E7. A well-studied effect of E6 is the degradation of tumor suppressor gene p53 via binding to the ubiquitin ligase E6AP. Additionally, E6 interacts with the cellular calcium-binding protein E6BP, but the functional significance remains unknown. Here we studied the effects of E6BP knockout in normal human and HPV-infected cell lines.

\section{Experimental Design or Project Methods:}

CRISPR-Cas9 system was used to knockout E6BP in HPV-human cervical cancer cell lines: HeLa (high-copy HPV18) and SiHa (low-copy HPV16) as well as human keratinocyte $\mathrm{HaCaT}$ (mutant p53) and p53-null lung carcinoma H1299 cell lines. Cells were transfected with a puromycin-resistant CRISPR ${ }^{*}$ Cas 9 control plasmid (pLentiv2) and one that carries a guide RNA against E6BP (pLentiv2-E6BP) and selected with puromycin. RFP-GFP, which does not carry a puromycin resistance, was transfected into cells to establish transfection efficiency and to serve as the control for the puromycin. After successful selection, polyclonal populations of pLentiv2 control and pLentiv2-E6BP cells were screened for E6BP protein levels. pLentiv2-E6BP cell populations with reduced $\mathrm{E} 6 \mathrm{BP}$ protein were serial diluted to generate monoclonal cell lines. Clones were then screened E6BP protein expression, and low expression clones were further processed for DNA sequencing of the E6BP gene editing site. Additionally, the effects of E6BP knockout in Siha and HeLa cells on p53, p21, and E6AP protein expression was studied by immunoblot.

\section{Results:}

Monoclonal pLentiv2-E6BP cell lines for H1299 and HaCaT cells were established and as well as polyclonal pLentiv2-E6BP HeLa and SiHa cell populations. Preliminary results indicate that E6BP knockout in HeLa and Siha cells reduces the p53 and p21 levels compared to pLentiv2 control cells. Additionally, E6AP protein expression was not affected by E6BP knockout in the polyclonal SiHa cells.

\section{Conclusion and Potential Impact:}

These preliminary results suggest that E6BP levels may influence p53 and p21 levels in HPV cells. It is of particular importance to further investigate if low levels of E6BP contribute to a worse outcome during HPV induced carcinogenesis. 